\title{
APR 271970
}

I. UNITED STATES

EPARTMENT OF

OMMERCE

UBLICATION

National Bureau of Standaros

Library, E-01 Admin. Bldg.
NBS TECHNICAL NOTE

391

\section{U. S. \\ IEPARTMENT \\ OF \\ COMMERCE \\ National \\ Bureau of Standards


The National Bureau of Standards ' was established by an act of Congress March 3, 1901. Today, in addition to serving as the Nation's central measurement laboratory, the Bureau is a principal focal point in the Federal Government for assuring maximum application of the physical and engineering sciences to the advancement of technology in industry and commerce. To this end the Bureau conducts research and provides central national services in four broad program areas. These are: (1) basic mcasurements and standards, (2) materials measurements and standards, (3) technological measurements and standards, and (4) transfer of technology.

The Bureau comprises the Institute for Basic Standards, the Institute for Materials Research, the Institute for Applied Technology, the Center for Radiation Rcsearch, the Center for Computer Sciences and Technology, and the Office for Information Programs.

THE INSTITUTE FOR BASIC STANDARDS provides the central basis within the United States of a complete and consistent system of physical measurement; coordinates that system with measurement systems of other nations; and furnishes essential services leading to accurate and uniform physical measurements throughout the Nation's scientific community, industry, and commerce. The Institute consists of an Office of Measurement Services and the following technical divisions:

Applied Mathematics-Electricity-Metrology-Mechanics-Heat-Atomic and Molecular Physics-Radio Physics "-Radio Engineering "-Time and Frequency "-Astrophysics "-Cryogenics."

THE INSTITUTE FOR MATERIALS RESEARCH conducts materials research leading to improved methods of measurement standards, and data on the properties of well-characterized materials needed by industry, commerce, educational institutions, and Government; develops, produces, and distributes standard reference materials; relates the physical and chemical properties of materials to their behavior and their interaction with their environments; and provides advisory and research services to other Government agencies. The Institute consists of an Office of Standard Reference Materials and the following divisions:

Analytical Chemistry_Polymers-Metallurgy_Inorganic Materials_Physical Chemistry.

THE INSTITUTE FOR APPLIED TECHNOLOGY provides tcchnical services to promote the use of available technology and to facilitate technological innovation in industry and Government; cooperates with public and private organizations in the development of technological standards, and test methodologies; and provides advisory and research services for Federal, state, and local government agencies. The Institute consists of the following technical divisions and offices:

Engineering Standards-Weights and Measures - Invention and Innovation - Vehicle Systems Research-Product Evaluation-Building Research-Instrument Shops--Measurement Engineering-Electronic Technology-Technical Analysis.

THE CENTER FOR RADIATION RESEARCH engages in research, measurement, and application of radiation to the solution of Bureau mission problems and the problems of other agencies and institutions. The Center consists of the following divisions:

Reactor Radiation-Linac Radiation-Nuclear Radiation-Applied Radiation.

THE CENTER FOR COMPUTER SCIENCES AND TECHNOLOGY conducts research and provides technical services designed to aid Government agencies in the selection, acquisition, and effective use of automatic data processing equipment; and servcs as the principal focus for the development of Federal standards for automatic data processing equipment, techniques, and computer languages. The Center consists of the following offices and divisions:

Information Processing Standards-Computer Information - Computer Services - Systems Development-Information Processing Technology.

THE OFFICE FOR INFORMATION PROGRAMS promotes optimum dissemination and accessibility of scientific information generated within NBS and other agencies of the Federal government; promotes the development of the National Standard Reference Data System and a system of information analysis centers dealing with the broader aspects of the National Measurcment System, and provides appropriate services to ensure that the NBS staff has optimum accessibility to the scientific information of the world. The Office consists of the following organizational units:

Office of Standard Reference Data-Clearinghouse for Fcderal Scientific and Technical Information "-Office of Technical Information and Publications-Library-Office of Public Information-Office of International Relations.

\footnotetext{
Headquarters and Laboratories at Gaithersburg. Maryland, unless otherwise noted: mailing address Washington, D.C, 20234 


\title{
UNITED STATES DEPARTMENT OF COMMERCE \\ Maurice H. Stans, Secretary
}

NATIONAL BUREAU OF STANDARDS Lewis M. Branscomb, Director

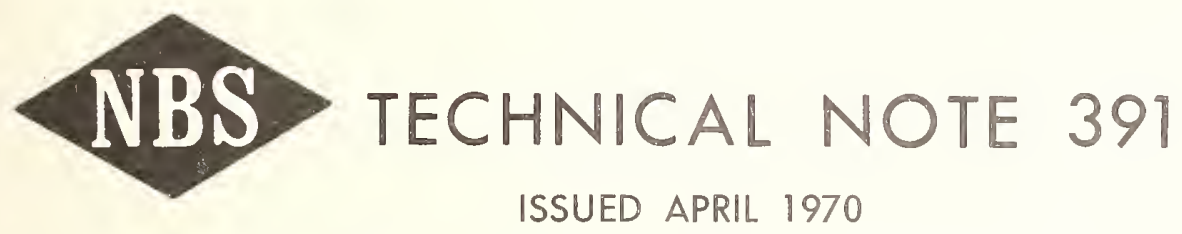

Nat. Bur. Stand. (U.S.), Tech. Note 391, 19 pages (April 1970) CODEN: NBTNA

\section{Quantifying Hazardous Microwave Fields: Analysis}

\author{
Paul F. Wacker \\ Electromagnetics Division \\ Institute for Basic Standards \\ National Bureau of Standards \\ Boulder, Colorado 80302
}

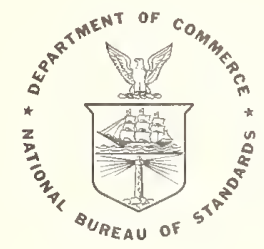

NBS Technical Notes are designed to supplement the Bureau's regular publications program. They provide a means for making available scientific data that are of transient or limited interest. Technical Notes may be listed or referred to in the open literature.

For sale by the Superintendent of Documents, U.S. Government Printing Office, Washington, D.C. 20402 (Order by SD Catalog No. C13.46:391, Price 30 cents 

ABSTRACT

I. Inadequacy of Accepted Concepts and Standards 2

II. Exposure Measurements in Waveguide and Coaxial Lines 4

III. Energy Density as a Measure of Hazard 5

IV. Measurement of Energy Density 6

V. Detailed Design of a Possible Probe 9

VI. Summary and Conclusions 14 


\section{LIST OF FIGURES}

Page

Fig. 1 Amplitude Variation Near a Horn-Lens Antenna

Fig. 2a Cross Section of Stark Cell

Fig. 2b General Plan of Stark Cell

18

Fig. 3 Cross Section of a Spherical Energy-Density Probe 
QUANTIFYING HAZARDOUS MICROWAVE FIELDS:

ANALYSIS

by

Paul F. Wacker

National Bureau of Standards

Boulder, Colorado

\section{ABSTRACT}

The familiar power density radiation hazard standards are quite satisfactory for a field consisting of a single infinite traveling plane wave. However, for microwave and lowe $r$-frequency fields, hazards occur primarily in near fields which cannot be approximated as the aforementioned plane wave. Further, power density can be quite misleading or even meaningless as a measure of hazard in a near field. Thus, power density in a standing wave can be precisely zero, yet the hazard of such a field can be arbitrarily large. Similarly, a reactive field may present a considerable hazard, yet have zerotime-average power density.

The major hazard from microwave and lower frequency radiation is believed to arise from dielectric heating of body tissues, and the heating of an isotropic medium is proportional to the sum of the squares of the absolute values of the electric field components $\left|E_{*}\right|^{2}+\left|E_{Y}\right|^{2}+\left|E_{Z}\right|^{2}$. Hence, electric field energy density is proposed for a radiation hazard standard.

Analytical limitations of various types of probes are considered and the advantages of a spherically-symmetric probe of lossy dielectric are discussed. For a rather general spherically-symmetric probe in an arbitrary field, both exact and approximate treatments are given for the calibration constant with full correction for the perturbation of the field by the probe. Conditions for a constant factor are also given.

Key Words: Hazards; Electromagnetic Radiation; Microwave Radiation; Radiation; Measurement; Standards; Energy Densitys Probe.

* This work was partially supported by the Bureau of Radiological Health. 
I. Inadequacy of Accepted Concepts and Standards.

For a linearly-polarized monochromatic infinite plane wave in a linear homogeneous isotropic medium, the magnitudes of the electric field, magnetic field, energy density, ${ }^{1}$ and energy flow ${ }^{1}$ (power density) are all readily measured and each of these magnitudes may be computed from any of the others without approximation, given the properties of the medium. Hence, any one of these magnitudes constitutes a valid index of such a field and, in fact, completely specifies the field apart from phase and the directions of propagation and polarization. Further, for such a wave in a lossless medium, only the phase varies with position or time. However, no such computation can be made for an arbitrary field. Rather, the ratios of these magnitudes can vary widely with the detailed nature of the field, i.e., depending upon detailed information seldom available in field monitoring or even in biological exposure experiments. For example, a standing wave formed from two of the preceding plane waves of equal amplitude and the same linear polarization but opposite direction has zero time-average energy flow (power density), yet the amplitudes of both the electric and magnetic fields are double those of the original waves at specific locations. Further, at distances from a source less than a small number of wavelengths (reactive zone), the time-average power flow may be zero, yet the electric and magnetic fields may be arbitrarily large.

${ }^{1}$ Strictly speaking, the familiar expressions for both energy density and energy flow (in terms of the electric and magnetic fields) can neither be proved nor disproved [J.A. Stratton, Electromagnetic Theory, McGrawHill, N.Y., N.Y., 1941, pp, 110, $131 \mathrm{ff.]}$. However, the expressions for energy absorption have strong foundations and "direct" measurements of energy density and energy flow are usually really measurements of energy absorption. Because the hazard discussed here is indeed related to the hypothetical energy density, we will use the expression energy density without qualification. 
The preceding complications are of little or no importance in the far field, i.e., for the usual practical sources, at distances from the source large compared to $\mathrm{a}^{2} / \lambda$ where $\lambda$ is the wavelength and $a$ is the greatest distance between points in the source, considering reflectors to be a part of the source. However, hazards and electromagnetic interference ${ }^{2}$ (EMI) arising from microwave and lower frequencies tend to be important in the near rather than the far field and a large fraction of biological exposure experiments are carried out under near field conditions to achieve high power levels.

"Measurements" of near fields based upon far field concepts must be regarded as simplistic and are at best semi-quantitative, often not even qualitatively correct. For example, in certain regions, the field arising from a microwave oven in a stainless steel kitchen may increase significantly with increasing distance from the oven. Even for a high gain antenna carefully designed to give a uniform field, the energy density may vary by a factor of two in a region of nominal constancy outside the reactive zone. The near electric field amplitude of such a horn-lens antenna 100 wavelengths on an edge is shown in fig. 1; the horizontal dependence is nominally that of a square wave and all the structure shown is real, not noise. Due to the wavelengths involved, analogies between microwaves and optics, $\mathrm{x}$-ray behavior, or gamma-ray behavior are commonly invalid and normally not as good as those with sound waves, since standing waves, resonances, and echoes occur with both microwaves and sound. Although workers in the field base their hazard measurements upon energy flow and commercial probes have energy flow scales, some of these probes respond primarily or partially to energy density per se.

2 Analytical considerations of electromagnetic interference parallel those of radiation hazards to a considerable extent. Thus, most of this paper also applies to EMI. 
II. Exposure Measurements in Waveguide and Coaxial Lines.

Perhaps the simplest non-far field situation applicable for exposure experiments with tissues or small animals is inside rectangular waveguide or coaxial line. Here the walls and inner conductor may be a copper mesh, fine compared to a wavelength. For waveguide, the largest inside dimension should be well between one-half and one wavelength so that one and only one mode will propagate. For lower frequencies, rectangular coaxial line such as that used in a Stark cell and shown in fig. 2 may be used; here the longest dimension should be significantly less than half a wavelength to prevent the propagation of more than one mode. If the tissue and its surrounding culture medium constitute a transverse slab which completely fills the cross-section of the guide or coaxial line and has a uniform complex dielectric constant, the field inside the sample may be readily computed from the dielectric constant and the input power. The field varies with the position in the cross-section and, if the slab is not thin compared to a waveguide, often with the longitudinal position as well. However, the longitudinal variation may be minimized by filling the guide with a medium having essentially the same complex dielectric constant and magnetic susceptibility as the slab, minimizing reflections. (In this case, the dimensions of the guide should be related to the wavelength in the medium and the medium should extend far enough on both sides of the tissue slab to insure a pure mode.) Further, attention may be confined to a region of uniform field. In the case of the Stark cell, high fields may be generated where the septum approaches the outside wall; to avoid contamination of the sample being studied by decomposition products from regions of high field, the septum should not be close to the wall and/or the portion of the sample being studied should be separated from the portion in the high field by a thin impervious dielectric. For a whole animal (invariably of irregular shape and varying dielectric constant), 
the situation is more complicated in any near field; however, in principle, reflection effects could also be minimized by immersion in a medium with a similar dielectric constant provided that gaps were small compared to a wavelength, that the medium did not cause too great an attenuation, and that thermal effects, respiration, etc. were properly handled.

\section{Energy Density as a Measure of Hazard.}

Since a field with initially zero energy flow (power density) may be responsible for arbitrarily great hazard to a subject later placed in it or arbitrarily great EMI to an electronic device placed in it, power density is not a suitable index of either hazard or EMI caused by a near field. Preferably the index should be closely related to the hazard and be easy to measure. Electrical energy density is proposed for the index. Since biological materials are essentially non-magnetic, they interact with an electromagnetic field primarily only because of their dipole moments (more strictly, the dipole transition moments), absorbing energy quanta at a rate proportional (for a linear medium) to the electrical energy density in the tissue. Although the quanta are much too small to cause the breaking of a chemical bond, a molecule which has absorbed one or more quanta has an activation energy which may well cause the molecule to be significantly more reactive in a general sense, say for exchange reactions involving the breaking "bonds" between compounds in the protoplasm and loosely held water. If desired, this may be considered to be extremely localized heating. Thus, electrical energy density should be a reasonable hazard index for both thermal and non-thermal effects including resonant absorption by specific molecules.

It is expected that the hazard to each portion of the body will be associated with its own "constant" of proportionality and that this 
"constant" will vary with frequency, perhaps sharply. There may well be other effects associated, e.g., with polarization of the field or nonlinearity of the tissue (say in pulsed fields). However, such effects can be studied as deviations from those predicted from energy density measurements assuming a linear isotropic medium.

For EMI in a near field, energy flow has the same disadvantages and energy density has many of the same advantages as for hazards.

\section{Measurement of Energy Density。}

The preceding energy density discussion is based upon the energy density within the given tissue, not upon that of the field into which the animal or person might enter. In principle, probes might be implanted in an animal or either the animal or person might be simulated in terms of the complex dielectric constant and magnetic susceptibility as a function of position. Since these procedures would be inconvenient for monitoring, the relation between the energy density inside an animal or person to that inside the probe must be considered (including perturbation of the field by the probe, person, or animal) and used in probe design. General principles will be discussed first, then illustrated with an exact treatment of a possible probe.

In principle, a probe should simulate the body or a given electronic instrument in its extraction of electrical energy from the field. Since the body makes only a slight perturbation in a field of hard $\mathrm{x}$-rays or gamma rays, so should a probe used to measure them, i.e., the probe should be analogous to a high resistance voltmeter rathe $r$ than a shorting ammeter which gives a reading strongly dependent upon the internal impedance of the source. In contrast, from the shortwave radio region through the ultraviolet, the body absorbs almost all the incident energy apart from that which it reflects. (The penetration depth ${ }^{3}$ increases steadily from $3000 \mathrm{GHz}$ to $3 \mathrm{MHz}$ with a depth of $10 \mathrm{~cm}$ for $30 \mathrm{MHz}$ and $52 \mathrm{~cm}$ for $3 \mathrm{MHz}$

3 Skin depth as defined in electromagnetics. 
[H.P. Schwan, "Survey of Microwave Absorption Characteristics of Body Tissues", in Proc. Second Tri-Service Conference on Biological Effects of Microwave Energy, July 8-10, 1958. ASTIA Document AD 131477 ]. Hence, a probe designed for this important frequency range should also absorb most of the non-reflected energy and probably have a low reflectivity as well [H.P. Schwan, "Radiation Biology, Medical Applications, and Radiation Hazards", in Microwave Power Engineering, vol 2, edited by Ernest C. Okress, Academic Press, New York, 1968 ]. (However, in some circumstances, say in continuous monitoring of a radar beam, it is impractical to use a probe which absorbs large quantities of energy.)

A related phenomenon concerns the magnetic field associated with the electric field. In absorption of a quantum of radiation, both magnetic and electric energy are involved. Further, both the initial electric and magnetic fields and the fields remaining after absorption are related by Maxwell's equations so that absorption of all the electric energy requires absorption of all the magnetic energy. For an infinite plane wave in a vacuum, the magnetic and electric energies are equal. However, the ratio of the energies may vary widely in a near field, say from the predominately magnetic field near an electromagnet to the predominantly electric field between plates of a large capacitor. Thus, a probe which properly simulates the body gives readings partially dependent upon the magnetic field, although the probability of quantum absorption is essentially independent of the magnetic field. For EMI measurements, a probe which reacts directly to the magnetic field may be desirable and is discussed in Section $V$ on probe design.

Any device which measures a single complex voltage or its magnitude may be regarded as a device which measures a single constituent of a field, i.e., the coefficient of a single basis function from 
the infinite series of basis functions used to represent the field. Thus, an ideal dipole measures the coefficient of one of the three electric dipole constituents of the field and completely neglects all magnetic multipoles and all but one of the electric multipole constituents of the field, regardless of their strengths. This limitation does not apply (in a practical sense) to a dipole in a linearly polarized plane wave only because the probe has a fixed calibration factor (which may be accurately computed); however, even in an infinite plane wave, a dipole oriented to give maximum amplitude can give an error of a factor of two in the energy density, depending upon whether the field is linearly or circularly polarized. A probe based upon losses (say, resulting in heating or pressure rise) does not have these limitations and so is suitable for direct measurement of total electric energy density; such a probe measures the energy density and adds all components without cancellation, all of the contributions being positive. For any electric (or magnetic) dipolar field, a probe which sums the squares of the absolute values of the voltages from three identical perpendicular dipoles can give an accurate result independent of probe orientation. Note that even an infinitesimal probe need not measure the field at a point, e.g., a quadrupolar probe; thus, a tiny dipole and a tiny quadrupole measure unrelated quantities.

A probe comparable in size to a wavelength will show resonances much the same as a half-wave dipole, i.e., the calibration constant will be a rather strong function of frequency, less so if the medium is lossy.

For convenience, a probe should not require orientation nor discriminate on the basis of direction of the incoming waves. It should be noted that no voltage probe has these properties, but that an energy density probe with spherical symmetry does. A general theory of such a probe is given in Section $V$, including a procedure for a priori computation of its calibration "constant", i.e., the ratio of the energy 
density of the field prior to the introduction of the probe to the energy density in the probe. No calibration constant can be computed for any probe without some knowledge or assumptions concerning the spatial dependence of the field, but an. energy density probe with spherical symmetry requires a minimum of knowledge - - much less than is required for a voltage probe.

For pulse power, the analysis is significantly more complicated. The present analysis has been modified to include pulse effects but will not be reported here.

V. Detailed Design of a Possible Probe.

Since previous studies have involved assumptions and approximations inappropriate for near fields, a detailed study is carefully carried out for a mathematically-manageable probe. The study reveals complications which can arise and illustrates some of the general statements of the preceding portions of this paper.

Consider a medium which is linear, isotropic, piecewise homogeneous, source-free, and subject to Ohm's law. For every homo= geneous region, every solution of Maxwell's equations is solenoidal and is a solution of the vector Helmholtz equation. For a single frequency and a medium which has spherical symmetry, the complex electric field $\underline{E}$ and magnetic field $\underline{H}$ are conveniently expressed as a linear combination of Hansen's $\underline{m}$ and $\underline{n}$ functions [Stratton, p. 416]. (The time-dependent factor $\mathrm{e}^{-\overline{\mathrm{i} \omega t}}$, where $\omega$ is the angular frequency, is assumed but suppressed.) Every such function is a solution of Maxwe11's equations [Stratton, p. 394], and the set of functions is presumably complete [Stratton, p. 393] for the problem at hand. (The I and 1 functions are not solutions of Maxwell's equations.)

Let $\mu$ and $\epsilon$ be the complex permeability and permittivity and $k=\omega(\mu \epsilon)^{\frac{1}{2}}$ be the propagation constant. As shown in Fig. 3, consider 
a probe with instrumentation from radius $R=0$ to a, a shield $S$ from $\mathrm{R}=\mathrm{a}$ to $\mathrm{b}$, a lossy material $\mathrm{L}$ from $\mathrm{R}=\mathrm{b}$ to $\mathrm{c}$, and a dielectric $\mathrm{D}$ used for thermal insulation from $R=c$ to $d$, immersed in a medium $A$, say air. If no internal instrumentation is used, $a$ and $b$ would be zero. Both the media and the propagation constants are indicated by the upper case Latin letters as shown in the diagram. The corresponding lower case Greek letters are used for the permeabilities. For an electrical probe, the permeabilities will ordinarily be essentially that of free space.

In the expression for the electric field, let $M_{\text {smnb }}$ and $N_{s m n b}$ be the coefficients of the ratios of $\underline{\mathrm{m}}_{\mathrm{smnb}}$ and $\underline{\mathrm{n}}_{\mathrm{Smnb}}$, respectively, to the square root of $n(n+1)(1+f) 2 \pi(n+m) ! /(2 n+1)(n-m) !$, where $f$ is unity for $\mathrm{m}=0$ but otherwise zero [Stratton, pp. 417-418]. The subscript $\mathrm{s}$ is used to denote the symmetry, either even (e) or odd (o). The subscript $\mathrm{b}$ indicates the kind of the spherical Bessel function, either the first (j) or second (y) kind. (The $\mathrm{y}^{\prime} \mathrm{s}$ are not needed for the central region of the probe ( $R=0$ to the first discontinuity) nor for the field in the absence of the probe.) The corresponding coefficients for $i \omega \mu \mathrm{H} / \mathrm{k}$ are $\mathrm{N}_{\text {smnb }}$ and $\mathrm{M}_{\text {smnb }}$ respectively (note reversal) [Stratton, p. 394].

Given the coefficients in any one region of the probe or the region outside the probe, the coefficients of the other regions can be obtained with the aid of boundary conditions [Stratton, pp. 483-4, (1a), (5), $(10)]$. Because of symmetry, coefficients with different s, m, or $n$ are independent, as are the $M$ and $N$ coefficients. Thus, for given $s$, $m$, and $n$, the four $M$ (or four $N$ ) coefficients corresponding to the two kinds of Bessel functions on the two sides of a boundary are related by two equations. Suppressing the $s, m$, and $n$ subscripts and adding a subscript to indicate the medium, the following equations are obtained: 


$$
\begin{aligned}
\left(N_{j A} j_{A}+N_{y A} y_{A}\right) A / \alpha & =\left(N_{j D} j_{D}+N_{y D} y_{D}\right) D / \delta \\
\left(N_{j A}{ }_{j A}+N_{y A} \partial_{y A}\right) / A & =\left(N_{j D} \partial_{j D}+N_{y D}{ }_{y D}\right) / D \\
M_{j A} j_{A}+M_{y A} y_{A} & =M_{j D} j_{D}+M_{y D} y_{D} \\
\left(M_{j A}{ }_{j A}+M_{y A}{ }_{y A}\right) / \alpha & =\left(M_{j D}{ }_{j D}+M_{y D}{ }_{y D}\right) / \delta
\end{aligned}
$$

for the A-D interface, where $j_{A}=j_{n}(A R), y_{A}=y_{n}(A R)$, and $\partial_{y D}=\frac{\partial}{\partial R}\left\{R j_{n}(D R)\right\}$, all here evaluated for $R=d$.

The coefficients of the field in the presence of the probe may be related to those for the field in the absence of the probe ("unperturbed field") by an integral equation, assuming that the perturbation of the field does not affect the distribution of the sources. (For a similar case, see Morse and Feshbach, Methods of Theoretical Physics, McGraw-Hill Book Co., N.Y., N.Y., 1953, pp. 1897, 1875.) Because of orthogonalities, the equation separates into a number of equations, each involving only $\mathrm{M}$ or $\mathrm{N}$ coefficients and a single set of $\mathrm{s}, \mathrm{m}$, and $\mathrm{n}$ values. Thus, a knowledge of the coefficients in any part of the probe, surrounding space, or in the unperturbed field permits determination of all the other coefficients, given the properties of the probe; further, determination of the coefficients splits into separate problems as indicated.

The time-average energy density is given by $(\underline{E} \cdot \underline{\bar{E}} \operatorname{Re} \epsilon+\underline{H} \cdot \underline{\bar{H}} \operatorname{Re} \mu / 4$, where the overline indicates that the complex conjugate is to be taken and the symbols $\underline{E}$ and $\underline{H}$ represent peak values. The integral of the timeaverage energy density over the polar and azimuthal angles $\theta$ and $\emptyset$ is simplified by the fact that the orthonormalities between the $\underline{m}$ 's and $\underline{n}^{\prime} s$ given by Stratton [ pp. 417-418] also apply for the Hermitian dot products 
(including those for solutions involving different kinds of Bessel functions if each square of a Bessel function in Stratton's expressions is replaced by the product of the first Bessel function by the complex conjugate of the second, both of the given order. For any medium, the integral of the time-average electric energy density over $\theta$ and $\emptyset$ is given by

$$
\begin{gathered}
\frac{R e \in}{4} \sum_{s, m, n}\left[\left|M_{s m n j} j_{n}+M_{s m n y} y_{n}\right|^{2}+\frac{n+1}{2 n+1} \mid N_{s m n j} j_{n-1}+N_{s m n y} y_{n-1}\right. \\
\left.+\frac{n}{2 n+1}\left|N_{s m n j} j_{n+1}+N_{\text {smny }} y_{n+1}\right|^{2}\right]
\end{gathered}
$$

and the integral of the time-average magnetic energy density is given by the expression obtained by interchanging the symbols $\mathrm{M}$ and $\mathrm{N}$ and replacing $\operatorname{Re} \epsilon$ by $\beta$. Here $\beta=|\mathrm{k} / \omega \mu|^{2} \operatorname{Re} \mu=|\epsilon / \mu| \operatorname{Re} \mu$; hence, for real $\mu, \beta=|\epsilon|$. The Bessel functions of course have the argument $k R$. The losses in a medium are given by an expression identical to that for the energy except that $\operatorname{Re} \epsilon$ is replaced by $2 \sigma$ and $\operatorname{Re} \mu$ is replaced by $2 x$, where $\sigma$ is the electrical conductivity and $x$ is the analogous magnetic quantity, both for the given frequency. Thus, $\operatorname{Re} \epsilon$ and $\beta$ are associated with the electrical and magnetic ene rgy density, while $\sigma$ and $X$ are associated with the electrical and magnetic losses respectively The possibility of a magnetic probe will be discussed late $r$.

Neither the unperturbed field nor, in the absence of internal instrumentation, the field in $\mathrm{L}$ involves $\mathrm{y}$ functions. Therefore, the timeaverage angular integrals of energy density in the unperturbed field and the loss in L depend only upon the sums

$$
\sum_{s, m}\left|M_{s m n j}\right|^{2} \text { and } \sum_{s, m}\left|N_{s m n j}\right|^{2} \text {, }
$$

not the individual coefficients. Further, the relations between th 
coefficients for the unperturbed field and those for $L$ are independent of both $s$ and $m$, so that specification of the sums for the unperturbed field determines the sums for L. Thus, neglecting losses in other regions, the calibration constant of the probe is independent of any changes in the unperturbed field which do not change the sums. A wide range applicability of the calibration "constant" is, of course, important for any probe, whether the calibration is computed or measured; such a range seems to be characteristic of the proposed probe.

It is, of course, convenient to be able to use and calibrate a probe in a plane wave and also to be able to specify the class of fields for which the calibration constant is applicable. For a plane wave travelling in the $z$ direction with its electric vector in the $x$ dirt ation, the $M_{\text {olnj }}$ 's are proportional to $i^{n}((2 n+1)(n+1) ! / n(n+1)(n-1) !)^{\frac{1}{2}}$, the $N_{\text {elnj }}$ s proportional to $-i$ times these quantities, and all the other coefficients zero [Stratton, p. 419]. Assuming that $\sum_{s, m}\left|M_{s m n j}\right|^{2}=\sum_{s, m}\left|I_{s m n j}\right|^{2}$ for each $\mathrm{n}$ requires that the electric and magnetic energies of the unperturbed field be equal if $\mathrm{Re} \epsilon=\beta$. Assuming in addition the sums to be zero for $n=0$ but proportional to $(2 n+1)(n+1) ! / n(n+1)(n-1)$ ! for other $n^{\prime}$ s permits one to compute a calibration constant (given the propagation constants and susceptibilities), valid for a large class of fields, including plane waves as a special case. (The calibration constant can, of course, also be obtained by direct measurement.). As will be discussed later, the se assumptions may be relaxed to a degree for a small probe with low dielectric constants.

Ordinarily the lossy material of the probe would have a complex permittivity and essentially a real permeability and so provide a measure of the electric field. 
It would be desirable to obtain an independent measure of the magnetic field, and this would be possible if a suitable material could be found, i.e., one which is linear and isotropic with a complex permeability but fairly small dielectric loss tangent. Such materials do exist for small fields (low on the hysteresis curve), but only exploratory work could determine whether a practical probe could be developed. The preceding analysis would apply for both kinds of probes, and provide information concerning the relative magnitudes of the $M$ and $N$ sums .

For a probe small compared to each of the $1 /|\mathrm{k}|$ 's, the Bessel functions may be approximated by $j_{n}(z) \cong z^{n} /(2 n+1)$ !! and $y_{n} \cong-(2 n-1) ! ! / z^{n+1}$, where $(2 n+1) ! !$ is the product of the odd integers up to and including $2 \mathrm{n}+1$. Hence, for the lower microwave frequencies and below, the preceding expressions are significantly simplified. Further, because practical fields have a limited amount of fine structure, at least the higher $M$ and $N$ coefficients tend to decrease with increasing $\mathrm{n}$, much like those for a plane wave. As a result, only the lower terms need be used for a probe tiny compared to both the scale of the field structure and a wavelength. (The wavelength condition is not required for $X$ - or $Y$-rays, where the wavelength is small compared to the dimensions of the probe, introducing averaging effects).

\section{Summary and Conclusions.}

The existing standards for mic rowave radiation hazards are based upon far-field concepts which may have little or even no validity in near fields. However, hazards occur largely in near fields, and even many dosimetry experiments are carried out in near fields. Because of the great complexity possible in a near field, a probe should, in principle, simulate the individual who might be subject to the possible hazard; however, such a probe is not feasible. Electric energy density 
provides a reasonable index of a large class of microwave radiation hazards and is simpler to measure in a near field than is energy flow. Analytical aspects of near field measurements are discussed in general, and design of a possible probe described in detail. So that near-field dosimetry experiments are meaningful and reproducible, great care must be used in field measurements and describing conditions which determine the field in the subject. 
100
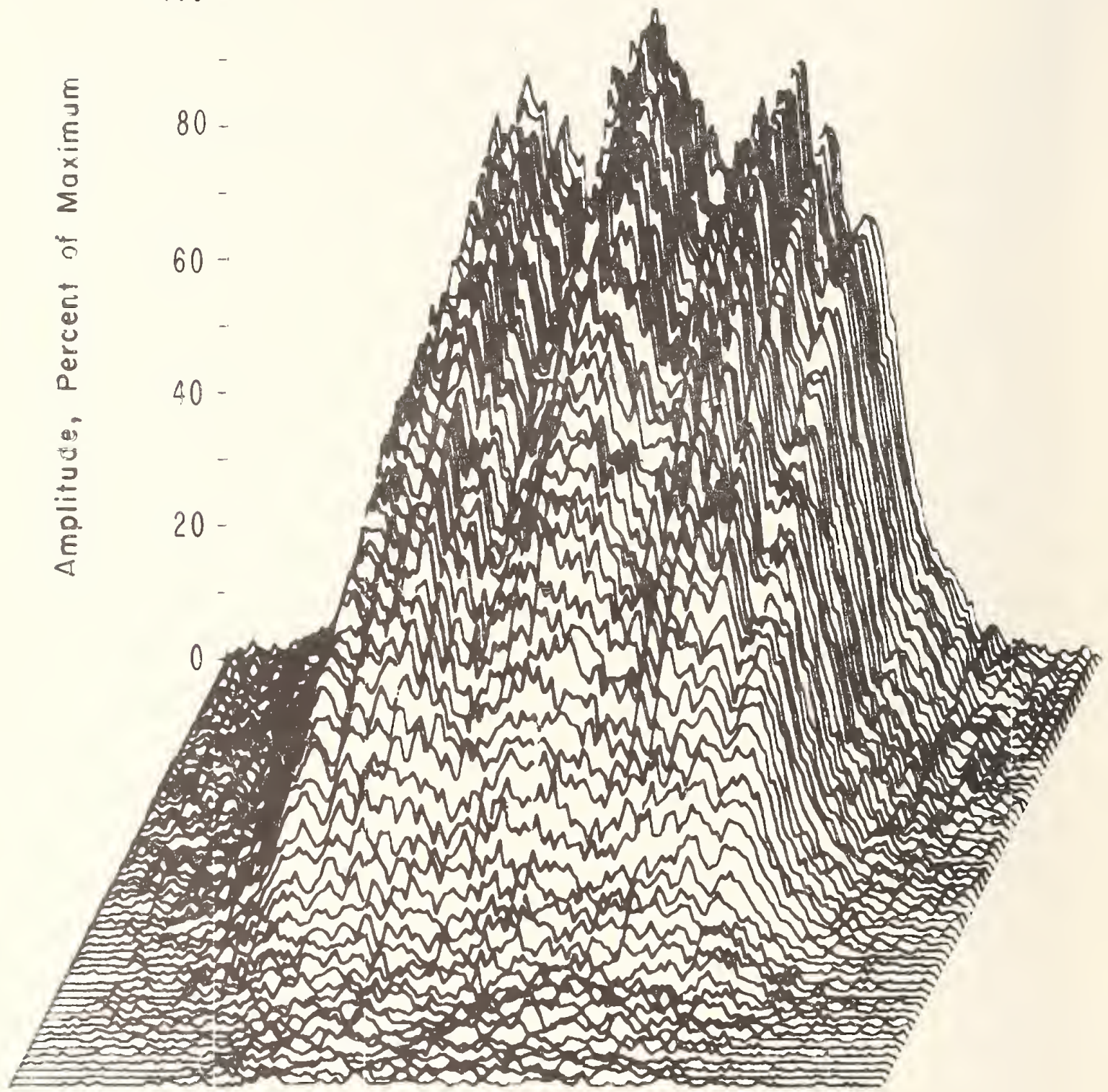

Horizontal $\longrightarrow$

Figure 1. Amplitude Variation Near a Horn-Lens Antenna. 


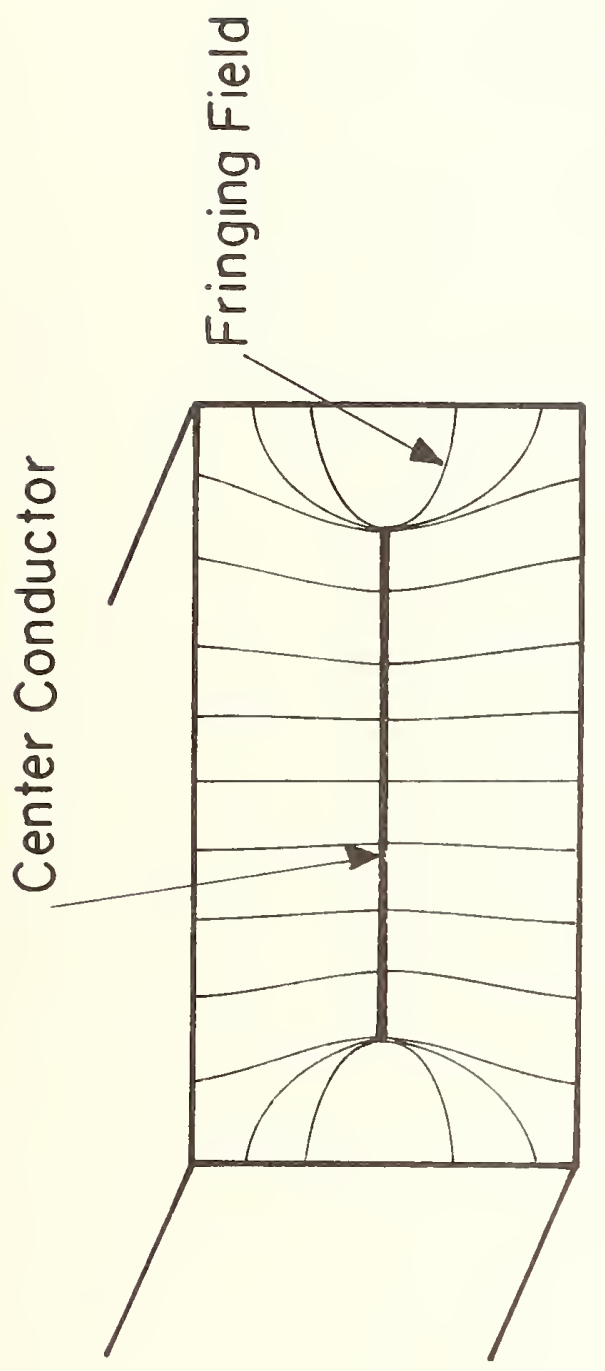

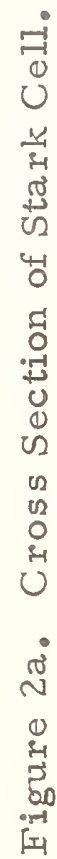




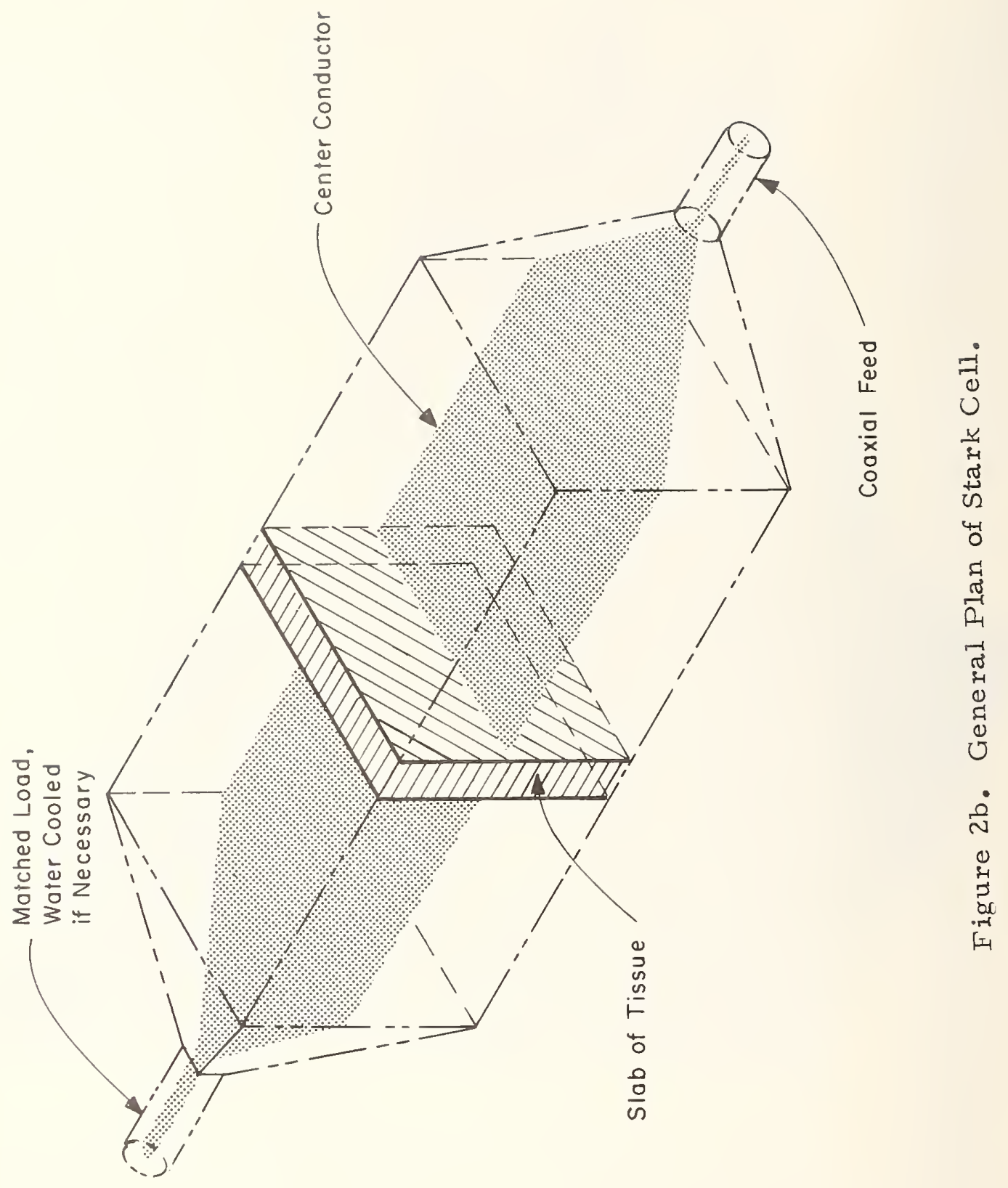




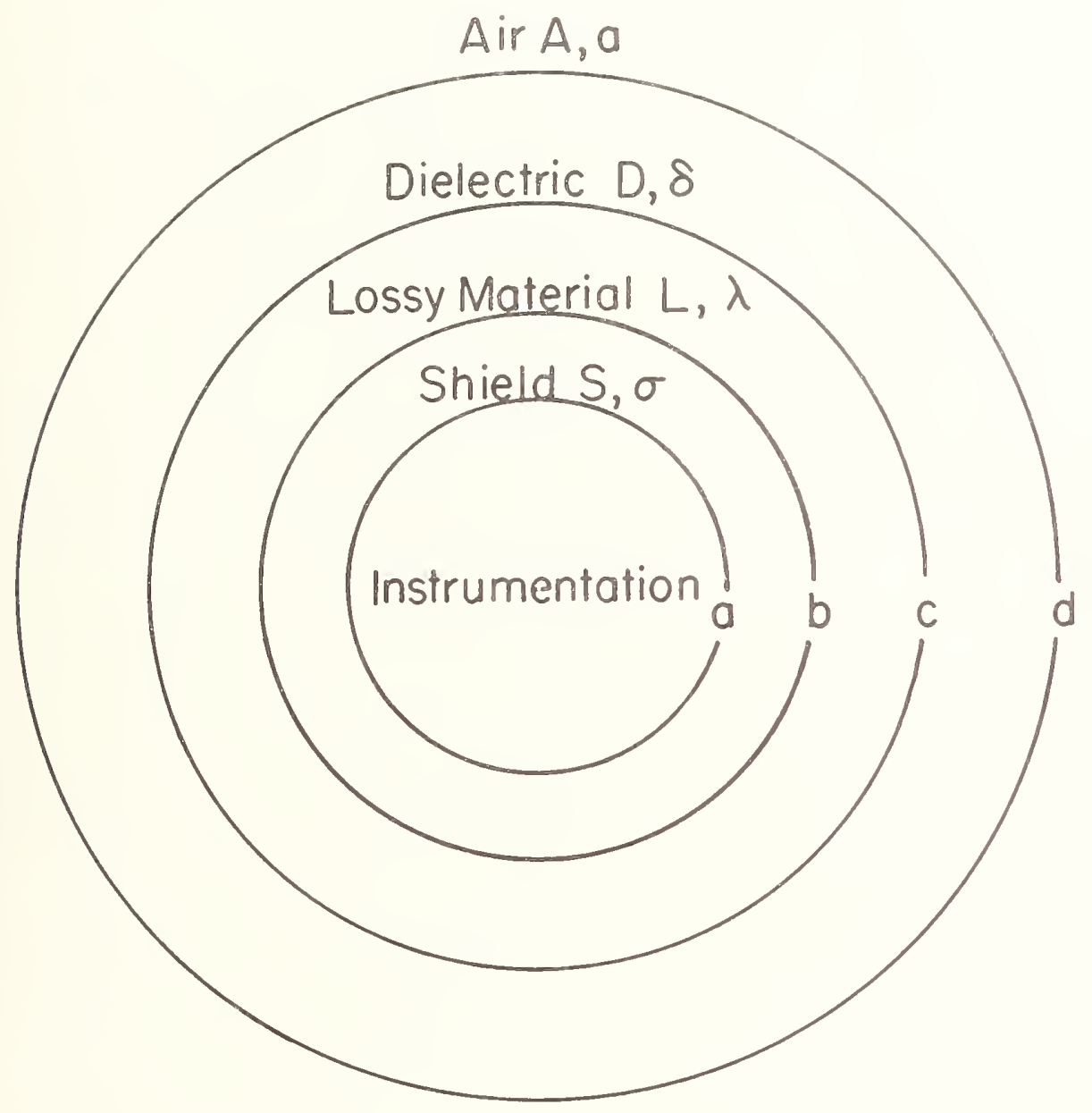

Figure 3. Cross Section of a Spherical Energy-Density Probe. 
Co 
PERIODICALS

JOURNAL OF RESEARCH reports National Bureau of Standards research and development in physics, mathematics, chemistry, and engineering. Comprehensive scientific papers give complete details of the work, including laboratory data, experimental procedures, and theoretical and mathematical analyses. Illustrated with photographs, drawings, and charts.

\section{Published in three sections, available separately:}

\section{Physics and Chemistry}

Papers of interest primarily to scientists working in these fields. This section covers a broad range of physical and chemical research, with major emphasis on standards of physical measurement, fundamental constants, and properties of matter. Issued six times a year. Annual subscription: Domestic, $\$ 9.50$; foreign, $\$ 11.75 *$.

\section{Mathematical Sciences}

Studies and compilations designed mainly for the mathematician and theoretical physicist. Topics in nathematical statistics, theory of experiment design, numerical analysis, theoretical physics and chemistry, logical design and programming of computers and computer systems. Short numerical tables. Issued quarterly. Annual subscription: Domestic, $\$ 5.00$; foreign, $\$ 6.25 *$.

\section{- Engineering and Instrumentation}

Reporting results of interest chiefly to the engineer and the applied scientist. This section includes many of the new developments in instrumentation resulting from the Bureau's work in physical measurement, data processing, and development of test methods. It will also cover some of the work in acoustics, applied mechanics, building research, and cryogenic engineering. Issued quarterly. Annual subscription: Domestic, $\$ 5.00$; foreign, $\$ 6.25 *$.

\section{TECHNICAL NEWS BULLETIN}

The best single source of information concerning the Bureau's research, developmental, cooperative and publication activities, this monthly publication is designed for the industry-oriented individual whose daily work involves intimate contact with science and technology-for engineers, chemists, physicists, research managers, product-development managers, and company executives. Annual subscription: Domestic, $\$ 3.00$; foreign, $\$ 4.00 *$.

- Difference in price is due to extra cost of foreign mailing.

\section{NONPERIODICALS}

Applied Mathematics Series. Mathematical tables, manuals, and studies.

Building Science Series. Research results, test methods, and performance criteria of building materials, components, systems, and structures.

Handbooks. Recommended codes of engineering and industrial practice (including safety codes) developed in cooperation with interested industries, professional organizations, and regulatory bodies.

Special Publications. Proceedings of NBS conferences, bibliographies, annual reports, wall charts, pamphlets, etc.

Monographs. Major contributions to the technical literature on various subjects related to the Bureau's scientific and technical activities.

National Standard Reference Data Series. NSRDS provides quantitive data on the physical and chemical properties of materials, compiled from the world's literature and critically evaluated.

Product Standards. Provide requirements for sizes, types, quality and methods for testing various industrial products. These standards are developed cooperatively with interested Government and industry groups and provide the basis for common understanding of product characteristics for both buyers and sellers. Their use is voluntary.

Technical Notes. This series consists of communications and reports (covering both other agency and NBS-sponsored work) of limited or transitory interest.

Federal Information Processing Standards Publications. This series is the official publication within the Federal Government for information on standards adopted and promulgated under the Public Law 89-306, and Bureau of the Budget Circular A-86 entitled, Standardization of Data Elements and Codes in Data Systems.

\section{CLEARINGHOUSE}

The Clearinghouse for Federal Scientific and Technical Information, operated by NBS, supplies unclassified information related to Government-generated science and technology in defense, space, atomic energy, and other national programs. For further information on Clearinghouse services, write:

Clearinghouse

U.S. Department of Commerce

Springfield, Virginia 22151 
U.S. DEPARTMENT OF COMMERCE

WASHINGTON, D.C. 20230

OFFICIAL BUSINESS

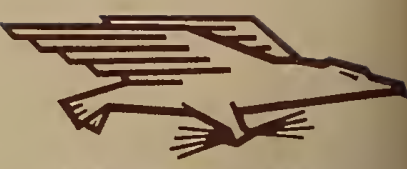

